\title{
Etiological spectrum of recurrent jaundice in adults: A retrospective observational study from a tertiary care center
}

\section{Gouranga Santra}

Department of Medicine, Midnapore Medical College, Paschim Medinipur, West Bengal, India

Address for the Correspondence:

Dr. Gouranga Santra Department of Medicine, Midnapore Medical College, Vidyasagar Road, Paschim Medinipur - 721 101,

West Bengal, India. E-mail: g.santra@yahoo.com

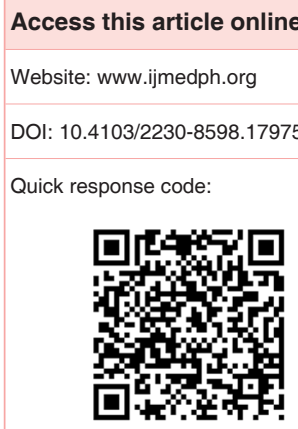

Introduction: Studies regarding etiological spectrum of recurrent jaundice are rare. We conducted this study to identify the causes of recurrent jaundice in a tertiary care center. Materials and Methods: Frequency of different causes of recurrent jaundice was assessed from 130 patients attended General Medicine Department over a period of 3 years. Recurrent jaundice was considered when patients had more than one episodes of jaundice with serum bilirubin $\geq 3 \mathrm{mg} / \mathrm{dl}$ since childhood. Recurrent jaundice was diagnosed from past medical records, records of follow-up visits, and current clinical presentation. Causes were identified from past and present medical records of history, clinical and laboratory examinations. Results: Causes of recurrent jaundice included prehepatic $(30 \%)$, hepatic $(59.23 \%)$, and posthepatic $(10.77 \%)$ disorders. Prehepatic disorders were Gilbert's syndrome (GS), megaloblastic anemia, autoimmune haemolytic anemia, Wilson's disease, G6PD deficiency, etc. Hepatic disorders were exacerbations of alcoholic hepatitis, hepatitis B and C, autoimmune hepatitis, congestive cardiac failure, sarcoidosis, benign recurrent intrahepatic cholestasis, eclampsia, pregnancy induced cholestasis, falciparum malaria, drug induced liver injury (DILI), etc. Posthepatic causes were choledocholithiasis, recurrent pancreatitis, periampullary carcinoma, choledochal cyst, ascariasis, hemobilia, HIV cholangiopathy, autoimmune pancreatitis, etc. Prehepatic jaundice cases were younger. The highest level of total bilirubin was seen in alcoholic hepatitis, DILI, and hepatitis B. Alcoholic hepatitis was the most common cause of recurrent jaundice $(19.23 \%)$. GS was the most common prehepatic cause. Mean age was lowest in GS and highest in DILI. Conclusion: Etiological spectrum of recurrent jaundice includes many prehepatic, hepatic or posthepatic disorders. A larger study may enlarge the spectrum.

Key words: Alcoholic hepatitis, congestive cardiac failure, drug induced liver injury, Gilbert's syndrome, jaundice

\section{INTRODUCTION}

Etiological diagnosis of recurrent jaundice is challenging because of the diversity of the causes. Recurrent jaundice has a wide spectrum of hematologic and hepatobiliary causes ranging from benign to life threatening. Causes of recurrent jaundice have not been tabulated in the literature. In this study, we have tried to evaluate and enumerate the etiological spectrum of the recurrent jaundice in adults in General Medicine Department in a tertiary care center.

This is an open access article distributed under the terms of the Creative Commons AttributionNonCommercial-ShareAlike 3.0 License, which allows others to remix, tweak, and build upon the work non-commercially, as long as the author is credited and the new creations are licensed under the identical terms.

For reprints contact: reprints@medknow.com

How to cite this article: Santra G. Etiological spectrum of recurrent jaundice in adults: A retrospective observational study from a tertiary care center. Int J Med Public Health 2016;6:35-9. 


\section{MATERIALS AND METHODS}

Frequency of different causes of recurrent jaundice was assessed from patients attended an out-patient clinic or admitted as in-patients in General Medicine Department over a period of 3 years from April 2011 to March 2014. Recurrent jaundice was considered when patients had more than one episodes of yellowish discoloration of eyes and urine and increased serum bilirubin level $(\geq 3 \mathrm{mg} / \mathrm{dl})$ since childhood. Patients would have intermittent normalization of bilirubin. Both spontaneous and drug induced recurrences were included in the study. Persistent clinical jaundice cases like thalassemia, $\mathrm{e} \beta$ thalassemia were excluded from the study.

Recurrent jaundice was diagnosed from past medical records, records of follow-up visits, and recent clinical presentation. The total number of cases presenting with recurrent jaundice during the study period was 130. Causes were identified from the past or present medical records of history regarding the risk factors and symptoms, physical examination findings, and laboratory investigations. Laboratory investigations were re-evaluated from records including liver function tests, complete blood count, serology for infectious hepatitis, abdominal imaging by ultrasonography or computed tomographic scanning, liver biopsy, magnetic resonance cholangiography (MRCP), and endoscopic retrograde cholangiopancreatography. Detailed evaluation of the cases was done routinely during their presentations. Informed consent was taken from the patients during evaluation of their records for including their data in the study.

\section{Statistical method}

Simple statistical methods are employed for the data analysis. Standard deviations, percentages (\%) and the ratio (:) are used to represent the data. GraphPad QuickCalcs online statistical calculator (GraphPad Software Inc., La Jolla, California, USA) is used for data analysis (http://www.graphpad.com/quickcalcs).

\section{RESULTS}

The mean age of patients was $34.56 \pm 12.03$ years. Male:female ratio of the patients was 78:52. Causes of recurrent jaundice in our study included prehepatic, hepatic, and posthepatic disorders [Table 1].

\begin{tabular}{|c|c|c|c|c|c|}
\hline Groups & $\begin{array}{l}\text { Causes of recurrent/ } \\
\text { intermittent jaundice }\end{array}$ & $\begin{array}{c}\text { Number of cases } \\
(n=130)\end{array}$ & Percentage & Male:female & $\begin{array}{l}\text { Highest total bilirubin } \\
\text { (mean) detected (mg/dl) }\end{array}$ \\
\hline \multirow{8}{*}{$\begin{array}{l}\text { Prehepatic } \\
39 \text { cases }(30 \%) \\
\text { Male:female-22:17 } \\
\text { Age- } 24.97 \pm 9.28 \text { years }\end{array}$} & Gilbert's syndrome & 15 & 11.54 & $8: 7$ & 4.9 \\
\hline & Vitamin B12 deficiency & 5 & 3.85 & $2: 3$ & 4.8 \\
\hline & Hematoma in hemophilia A & 3 & 2.31 & $3: 0$ & 4.2 \\
\hline & Hematoma in hemophilia B & 1 & 0.77 & $1: 0$ & 4.1 \\
\hline & Wilson's disease & 7 & 5.38 & $4: 3$ & 7.6 \\
\hline & AlHA & 4 & 3.08 & $1: 3$ & 4.9 \\
\hline & $\begin{array}{l}\text { Drugs causing hemolysis with } \\
\text { G6PD deficiency }\end{array}$ & 3 & 2.31 & $3: 0$ & 4.7 \\
\hline & Sickle cell crisis & 1 & 0.77 & $0: 1$ & 8.9 \\
\hline \multirow{14}{*}{$\begin{array}{l}\text { Hepatic } \\
77 \text { cases }(59.23 \%) \\
\text { Male:female- } 49: 28 \\
\text { Age- } 38.90 \pm 11.09 \text { years }\end{array}$} & Sarcoidosis & 2 & 1.54 & $1: 1$ & 7 \\
\hline & Alcoholic hepatitis & 25 & 19.23 & $23: 2$ & 13.8 \\
\hline & Falciparum malaria & 2 & 1.54 & $1: 1$ & 8.3 \\
\hline & Hyperemesis gravidarum & 1 & 0.77 & $0: 1$ & 4.8 \\
\hline & Preeclampsia/eclampsia & 2 & 1.54 & $0: 2$ & 4.2 \\
\hline & Exacerbations of hepatitis $B$ & 12 & 9.23 & $7: 5$ & 12.3 \\
\hline & Exacerbations of hepatitis $C$ & 2 & 1.54 & $1: 1$ & 8.7 \\
\hline & Autoimmune hepatitis & 3 & 2.31 & $1: 2$ & 8.9 \\
\hline & Hepatitis B+AlHA & 1 & 0.77 & $0: 1$ & 13 \\
\hline & PSC & 1 & 0.77 & $1: 0$ & 6.4 \\
\hline & CCF with decompensations & 12 & 9.23 & $7: 5$ & 5.2 \\
\hline & BRIC & 2 & 1.54 & $1: 1$ & 10.4 \\
\hline & $\mathrm{ICP}$ & 1 & 0.77 & $0: 1$ & 4.7 \\
\hline & Drug induced liver injury & 11 & 8.46 & $6: 5$ & 15.3 \\
\hline Posthepatic & Gall stone in CBD & 4 & 3.08 & $1: 3$ & 10.7 \\
\hline 14 cases $(10.77 \%)$ & Periampullary carcinoma & 1 & 0.77 & $1: 0$ & 8.9 \\
\hline Male:female-7:7 & Relapsing pancreatitis & 3 & 2.31 & $1: 2$ & 4.5 \\
\hline \multirow{5}{*}{ Age $-37.43 \pm 8.28$ years } & Autoimmune pancreatitis & 1 & 0.77 & $1: 0$ & 13 \\
\hline & Choledochal cyst & 1 & 0.77 & $0: 1$ & 7.9 \\
\hline & Ascariasis & 2 & 1.54 & $1: 1$ & 5.8 \\
\hline & Hemobilia with HCC & 1 & 0.77 & $1: 0$ & 6.9 \\
\hline & HIV cholangiopathy & 1 & 0.77 & $1: 0$ & 9.6 \\
\hline
\end{tabular}

$\mathrm{CCF}=$ Congestive cardiac failure, ICP = Intrahepatic cholestasis of pregnancy, AlHA = Autoimmune hemolytic anemia, BRIC = Benign recurrent intrahepatic cholestasis, $\mathrm{CBD}=$ Common bile duct, $\mathrm{HCC}=$ Hepatocellular carcinoma, $\mathrm{PSC}=$ Primary sclerosing cholangitis 
Prehepatic causes of recurrent jaundice in our study included Gilbert's syndrome (GS), drug-induced hemolysis with or without G6PD deficiency, autoimmune hemolytic anemia (AIHA), hemophilia A and B, Wilson's disease (WD), and Vitamin B 12 deficiency (VBD). Fifteen cases of GS were detected with intermittent jaundice due to fasting, stress or intercurrent illness. Except jaundice, they had no other clinical findings. Recurrent hemolysis in three G6PD deficient patients on exposure to dapsone, cotrimoxazole, and nitrofurantoin (one case of each) caused recurrent jaundice. Seven of our patients had jaundice with hemolysis due to WD with past h/o similar episodes. Recurrent hematoma formation with hemophilia A and B was detected with recurrent mild jaundice in four cases. Intermittent mild jaundice was noted in five cases with VBD. Four cases had AIHA, and they were found to be positive for direct Coomb's test and the antinuclear factor was positive in one case.

Two of our patients had recurrent cholestatic jaundice with sarcoidosis. Spontaneous exacerbation of hepatitis B and C were detected to cause recurrent hepatocellular jaundice in 12 and two cases, respectively. Exacerbations of alcoholic hepatitis were detected in 25 cases who failed abstinence. Three cases of autoimmune hepatitis $(\mathrm{AIH})$ were detected with intermittent jaundice. Two falciparum malaria cases with hepatocellular jaundice had a relapse of the disease. Both AIHA and viral hepatitis B were seen in one case with intermittent jaundice due to either exacerbation of disease activity of AIHA or hepatitis B. We had one case of recurrent jaundice due to primary sclerosing cholangitis (PSC).

Congestive cardiac failure (CCF) cases were detected with episodes of mild jaundice during cardiac decompensation in 12 cases. Benign recurrent intrahepatic cholestasis (BRIC) was seen in two cases. Four patients had recurrent jaundice in subsequent pregnancies, like one case of intrahepatic cholestasis of pregnancy (ICP) in the third trimester, one case of hyperemesis gravidarum in first trimester, two cases of preeclampsia/eclampsia with mild to moderately increased bilirubin level. Jaundice resolved after the delivery and reappeared in a subsequent pregnancy.

Rechallenge of antitubercular drugs (ATDs) was responsible for five cases of recurrent hepatocellular jaundice. Amoxycillin-clavulanic acid was responsible for drug-induced liver injury (DILI) in one case with hepatocellular and followed by cholestatic jaundice with recurrence on re-exposure. Oral contraceptive pill (OCP) was responsible for one case of recurrent cholestatic jaundice. Amitriptyline and valproate were responsible for one case each of hepatocellular injury with recurrence on re-exposure. Herbal medicines were found in two cases of recurrent hepatocellular jaundice.

Posthepatic causes included gallstone in the common bile duct (CBD) in four cases and relapsing pancreatitis in three cases. Hemobilia with recurrent jaundice was seen in one case with hepatocellular carcinoma (HCC). Ascaris in CBD with recurrent jaundice was seen in two of our patients. Recurrent HIV cholangiopathy was seen in one patient with intermittent jaundice. We had one young female with choledochal cyst with dilatation of extrahepatic duct. She had recurrent jaundice associated with cholangitis. Periampullary carcinoma was seen in one case.

Patients with prehepatic recurrent jaundice were younger than patients with hepatic and posthepatic causes. Mean ages of the common disorders of recurrent jaundice included $24.40 \pm 8.66$ years in GS, $37.80 \pm 9.60$ years in VBD, $25 \pm 7.77$ years in WD, $35.36 \pm 6.43$ years in alcoholics, $43.92 \pm 13.43$ in CCF patients, $51.18 \pm 7.76$ years in DILI, and $38.25 \pm 9.96$ years in hepatitis B. Mean age was lowest in GS patients and highest in DILI patients. The level of total bilirubin (mean) was relatively higher ( $>12 \mathrm{mg} / \mathrm{dl}$ ) during exacerbations in alcoholic hepatitis, hepatitis B, and DILI patients.

\section{DISCUSSION}

Recurrent jaundice is a diagnostic challenge to physicians. After extensive evaluation, the causes of recurrent jaundice remain obscure in a large number of cases. Causes of recurrent jaundice in our study included both medical and surgical disorders and distribution of causes included prehepatic (30\%), hepatic $(59.23 \%)$, and posthepatic $(10.77 \%)$ disorders. Alcoholic hepatitis was the most common cause of recurrent jaundice, followed by GS.

In GS mild jaundice appears under conditions of exertion, stress, fasting, intercurrent illness, dehydration, menstruation, and lack of sleep. Jaundice disappears when these conditions resolve. In our study, GS was detected to be the most common prehepatic cause of recurrent jaundice (11.54\% cases). VBD can cause recurrent jaundice due to ineffective erythropoiesis as seen in our study. ${ }^{[1,2]}$ Resorption of recurrent large hematomas caused recurrent mild jaundice with unconjugated hyperbilirubinemia in hemophilia A and B. Jaundice in WD disease is due to hemolysis. Excess generation of free radicals by copper toxicity in WD causes recurrent hemolysis. Jaundice in WD may also occur due to hepatocellular involvement. WD patients in our study had recurrent jaundice due to episodes of hemolysis. Hemolytic anemias like G6PD deficiency, AIHA, etc., were also seen with a recurrence of hemolysis and jaundice. Recurrent hemolytic jaundice with G6PD deficiency occurred with re-exposure to drugs like dapsone, cotrimoxazole, and nitrofurantoin.

Episodes of decompensation of CCF can cause intermittent mild jaundice with approximately $50-60 \%$ of bilirubin being unconjugated as a result of mild hemolysis, reduced uptake, and decreased conjugation by hepatocytes. Serum bilirubin may fall rapidly after improvement of hepatic congestion, however in prolonged heart failure bilirubin level may take months to become normal after relief of hepatic congestion due to the formation of delta bilirubin ( $\mathrm{T}^{1 / 2} 21$ days) (covalent bonding of conjugated bilirubin with albumin). CCF was an important cause (9.23\% cases) of intermittent mild jaundice in our study due to episodes of the cardiac decompensation.

Sarcoidosis can cause intermittent cholestatic jaundice due to fluctuating disease activity as seen in our study. ${ }^{[3]}$ Chronic alcoholic 
liver disease patients who fail abstinence may develop episodes of acute alcoholic hepatitis. ${ }^{[4]}$ Alcoholic hepatitis was the most common cause of recurrent jaundice in our study due to persistent alcohol consumption (19.23\% cases). Intermittent jaundice due to spontaneous acute exacerbation of chronic hepatitis B is not uncommon. ${ }^{[5-7]}$ Acute exacerbation of chronic hepatitis $C$ is also seen. ${ }^{[8,9]}$ Recurrent jaundice due to hepatitis $\mathrm{B}$ was 6 times more common than the hepatitis $C$ in our study. Recurrent jaundice cases were also seen due to recrudescence or reinfection of falciparum malaria.

DILI can cause recurrent jaundice on re-exposure to drugs, and liver function tests may have a necroinflammatory, cholestatic, or a mixed pattern. Recurrent hepatocellular jaundice occurs with rechallenge of ATDs (pyrazinamide, rifampicin, and isoniazid) in about $11 \%$ cases. ${ }^{[10]}$ In our study rechallenge of ATDs was a major cause of DILI. Amoxicillin-clavulanic acid also caused recurrent DILI on re-exposure. Amitriptyline, valproate, and herbal medicines can cause recurrent hepatocellular jaundice as seen in our study. OCP was found to cause recurring cholestatic jaundice.

Recurrent jaundice can occur in subsequent pregnancies due to ICP in second or third trimester, hyperemesis gravidarum in first trimester, preeclampsia/eclampsia in third trimester or HELLP syndrome (hemolysis, elevated liver enzymes, low platelet count) in third trimester. ${ }^{[1]}$ Though ICP, preeclampsia/eclampsia and hyperemesis gravidarum caused recurrent jaundice in our study, HELLP syndrome was not seen.

BRIC is responsible for recurrent cholestatic jaundice with spontaneous improvement as seen in two of our cases. PSC is a disease of the bile ducts that causes inflammation and subsequent obstruction of the bile ducts both at an intrahepatic and extrahepatic level. Intermittent jaundice in PSC is due to the recurrent bacterial cholangitis. ${ }^{[12]}$ The single case of PSC in our study had preceding $\mathrm{h} / \mathrm{o}$ ulcerative colitis and diagnosed with MRCP showing multifocal strictures and segmental dilatation. Intermittent jaundice was seen in AIH cases due to the fluctuation of disease activity. Acute or chronic relapsing pancreatitis can cause a recurrence of cholestatic jaundice. ${ }^{[13]}$ Two female and one male case of relapsing pancreatitis in our study were due to cholelithiasis and alcohol consumption, respectively. Autoimmune pancreatitis (AIP) and IgG4 related disease can cause recurrent jaundice during its natural course or steroid tapering or withdrawal. ${ }^{[14]}$ A case of AIP in our study developed intermittent cholestatic jaundice during its natural course.

Choledocholithiasis was an important posthepatic cause of recurrent jaundice. Complete obstruction of CBD by stone may be intermittent, so patients may report episodic jaundice. Cholangitis is relatively frequent in-patients with a benign biliary obstruction like choledocholithiasis, in contrast to malignant obstruction. Recurrent cholangitis was the cause of recurring jaundice in our two cases with choledocholithiasis. Ascaris can cause jaundice by entering sphincter of Oddi and causing obstruction, but it flees from the site due to contact with irritant bile relieving the obstruction, but there may be reinvasion, so causing fluctuating obstructive jaundice. ${ }^{[15]}$ Invasion of the CBD with ascaris may also cause cholangitis. ${ }^{[15]}$ One case of ascaris in CBD in our study was associated with recurrent cholangitis. Secondary sclerosing cholangitis due to opportunistic infection is increasingly seen in immunodeficient patients with HIV. Cryptosporidium parvum, cytomegalovirus, and microsporidia are the organisms most frequently found. HIV cholangiopathy is associated with intra or extrahepatic sclerosing cholangitis, biliary stricture, or papillary stenosis. HIV cholangiopathy case in our study had intermittent jaundice due to superadded bacterial cholangitis.

Liver cysts and tumors can cause recurrent jaundice. ${ }^{[16,17]}$ Jaundice is usually mild and transitory in congenital liver cyst due to intermittently increased pressure on bile ducts, probably due to intracystic hemorrhage. ${ }^{[16]}$ Liver cysts and tumors causing recurrent jaundice were not found in our study. Choledochal cyst is commonly a disease of childhood, but it can also present in adults especially in the female. The choledochal cyst may present with recurrent jaundice due to cholangitis. It may also present with jaundice and/or abdominal mass without cholangitis. Recurrent jaundice in our patient was due to cholangitis. Duodenal diverticulum and intrahepatic cholangiocarcinoma are reported to cause intermittent obstructive jaundice though we have not found any such cases. ${ }^{[18-20]}$ Ampullary tumors may produce intermittent jaundice because of sloughing of the tumor and partial relief of the block, as seen in one case of periampullary carcinoma in our study. Hemobilia, mostly a result of hepatic trauma or neoplasm, presents with the triad of biliary colic, jaundice, and gastrointestinal bleeding. HCC may occasionally present with recurrent hemobilia and jaundice. ${ }^{[21]}$ One of our patient of HCC also had recurrent hemobilia and jaundice.

\section{CONCLUSION}

Jaundice may be recurrent in many prehepatic, hepatic, or posthepatic disorders. Alcoholic hepatitis and GS are the most common causes of recurrent jaundice in our study. Frequency of different causes in this study does not represent the general population as it was done in a tertiary care center and etiological spectrum detected is also not complete as it is a limited study. A larger study is needed to enlarge the etiological spectrum.

\section{Financial support and sponsorship}

Nil.

\section{Conflicts of interest}

There are no conflicts of interest.

\section{REFERENCES}

1. Joshi D, Kumar N, Rai A. Recurrent reversible jaundice, neuropsychiatric manifestations, dementia and ataxia as manifestations of vit. B12 deficiency. J Assoc Physicians India 2008;56:475-6.

2. Dasari S, Naha K, Prabhu M. An unusual cause for recurrent jaundice in an otherwise healthy male. Australas Med J 2012;5:541-3. 
3. Bandopadhyay R, Santra G, Das I, Mukherjee T. Sarcoidosis presenting as recurrent jaundice. J Assoc Physicians India 2014;62:655-6.

4. O'Shea RS, Dasarathy S, McCullough AJ. Alcoholic liver disease. Am J Gastroenterol 2010;105:14-32.

5. Sarin SK, Kumar A, Almeida JA, Chawla YK, Fan ST, Garg H, et al. Acute-on-chronic liver failure: Consensus recommendations of the Asian Pacific Association for the study of the liver (APASL). Hepatol Int 2009;3:269-82.

6. Lok AS, Lai CL. Acute exacerbations in Chinese patients with chronic hepatitis $B$ virus (HBV) infection. Incidence, predisposing factors and etiology. J Hepatol 1990;10:29-34.

7. Wong VW, Chan HL. Severe acute exacerbation of chronic hepatitis B: A unique presentation of a common disease. J Gastroenterol Hepatol 2009;24:1179-86.

8. Wong RJ, Cheung RC. Acute exacerbation among chronic hepatitis $\mathrm{C}$ patients: Tip of the iceberg that deserves more attention. Clin Gastroenterol Hepatol 2013;11:1181-2.

9. Sagnelli E, Pisaturo M, Stanzione M, Messina V, Alessio L, Sagnelli C, et al. Clinical presentation, outcome, and response to therapy among patients with acute exacerbation of chronic hepatitis C. Clin Gastroenterol Hepatol 2013;11:1174-1180.e11.

10. Sharma SK, Singla R, Sarda P, Mohan A, Makharia G, Jayaswal A, et al. Safety of 3 different reintroduction regimens of antituberculosis drugs after development of antituberculosis treatment-induced hepatotoxicity. Clin Infect Dis 2010;50:833-9.

11. Larrey D, Rueff B, Feldmann G, Degott C, Danan G, Benhamou JP. Recurrent jaundice caused by recurrent hyperemesis gravidarum. Gut 1984;25:1414-5.
12. Hawkes ND, Mutimer D, Thomas GA. Intermittent jaundice and rigors in a patient with longstanding ulcerative colitis. Postgrad Med J 2001;77: 406-7, 412-3.

13. Abdallah AA, Krige JE, Bornman PC. Biliary tract obstruction in chronic pancreatitis. HPB (Oxford) 2007;9:421-8.

14. Hirano K, Tada M, Isayama H, Yagioka H, Sasaki T, Kogure H, et al. Longterm prognosis of autoimmune pancreatitis with and without corticosteroid treatment. Gut 2007;56:1719-24.

15. Khuroo MS, Zargar SA, Mahajan R. Hepatobiliary and pancreatic ascariasis in India. Lancet 1990;335:1503-6.

16. Santman FW, Thijs LG, Van Der Veen EA, Den Otter G, Blok P. Intermittent jaundice: A rare complication of a solitary nonparasitic liver cyst. Gastroenterology 1977;72:325-8.

17. Beretta E, De Franchis R, Staudacher C, Faravelli A, Primignani M Vecchi $\mathrm{M}$, et al. Biliary cystadenoma: An uncommon cause of recurrent cholestatic jaundice. Am J Gastroenterol 1986;81:138-40.

18. Alvarez Sánchez A, Rivero Cruz MA, Ladero Quesada JM, Moreno Tello B, Díaz-Rubio M. Intermittent obstructive jaundice as a form of presentation of duodenal diverticulum. An Med Interna 1999;16: 193-5.

19. Capizzi PJ, Rosen CB, Nagorney DM. Intermittent jaundice by tumor emboli from intrahepatic cholangiocarcinoma. Gastroenterology 1992; 103:1669-73.

20. Fischer CP, Fernandez M, Garden OJ. Papillary cholangiocarcinoma: A cause of intermittent, obstructive jaundice. Surgery 2002;131:234-5.

21. Hsu CL, Wang $\mathrm{CH}$, Chen RJ, Chen TC. Hepatocellular carcinoma presenting as jaundice, hemobilia, and acute pancreatitis: A case report Changgeng Yi Xue Za Zhi 1998;21:232-6. 